\title{
Three Cases With a Lung Abscess and Bronchial Inflammatory Polyps
}

\author{
Yoshiyuki Kurose ${ }^{a}$, Norio Kodaka ${ }^{a}$, Kumiko Kashiwa ${ }^{a}$, Yuuki Kamata ${ }^{a}$, Haruhi Kouno ${ }^{a}$, Chihiro Nakano, \\ Kayo Sugawa, Testuro Sawata ${ }^{a}$, Atsuo Miura ${ }^{a}$, Toru Yamagishia, Nobuyuki Hatanaka ${ }^{a}$, \\ Hirotaka Kamemura ${ }^{\text {a }}$, Asako Kitahara ${ }^{\text {a }}$, Yuki Yokouchib ${ }^{\text {, }}$ Ken Sanno ${ }^{\text {a, c }}$
}

\begin{abstract}
Three patients who had a lung abscess and bronchial inflammatory polyps are presented. The patients, all males, were 45,65 , and 74 years old. Imaging studies showed a lung abscess, and bronchoscopy revealed protruding lesions in the involved bronchi. The histological findings were inflammatory polyps. In all cases, the inflammatory polyps regressed with systemic antibiotic treatment, and the lung abscess improved. These cases were interesting in terms of the etiology of bronchial inflammatory polyps.
\end{abstract}

Keywords: Benign inflammatory polyps; Bronchial inflammatory polyp; Bronchial stenosis; Lung abscess

\section{Introduction}

Bronchial inflammatory polyps are relatively rare. They are inflammatory protruding lesions that develop mainly in the trachea and proximal bronchi from secondary bronchus [1]. Three patients with a lung abscess and bronchial inflammatory polyps in which improvement was achieved with systemic antibiotic treatment are presented.

\section{Case Report}

\section{Case 1}

A 74-year-old man was evaluated for a productive cough

\footnotetext{
Manuscript accepted for publication November 3, 2010

${ }^{a}$ Department of Respiratory Medicine, Toho University Ohashi Medical Center, Tokyo, Japan

${ }^{\mathrm{b}}$ Department of Pathology, Toho University Ohashi Medical Center, Tokyo, Japan

${ }^{\mathrm{c} C o r r e s p o n d i n g ~ a u t h o r: ~ 2-17-6 ~ O h a s h i, ~ M e g u r o-k u, ~ T o k y o, ~ 153-8515, ~}$

Japan. Email: sken@oha.toho-u.ac.jp
}

doi:10.4021/jmc74w with hemosputa. Chest X-ray showed an infiltrative shadow with cavity formation in the right mid-lung field, he was admitted to hospital. His past medical history included chronic sinusitis and nasal polyp surgery. On physical examination at admission, the patient was afebrile, and auscultation revealed no abnormalities. Blood tests showed a WBC count of 16,900 (seg $81.5 \%$ ) and CRP of $7.7 \mathrm{mg} / \mathrm{dl}$, indicating an increased inflammatory reaction. Sputum cultures revealed no significant pathogens. Chest CT (Fig. 1A) showed a lung abscess in right S3. Bronchoscopic findings (Fig. 2A) were a protruding lesion at the opening of right B3, with stenosis of the involved bronchus.

The histological findings obtained from bronchial biopsy (Fig. 3) were extensive neutrophilic infiltration in the submucosal tissue. In some areas, there was inflammatory cell infiltration together with fibroblast proliferation, but there was no evidence of malignancy. The diagnosis was a bronchial inflammatory polyp. BIPM $0.6 \mathrm{mg}$ was administered by intravenous infusion for 16 days. After treatment (about 4 months later), Chest CT (Fig. 1B) showed a cavitary lesion, but the fluid accumulation resolved, so the lung abscess had improved. A bronchoscopy on day 24 (Fig. 2B) was disappearance of the protruding lesion at the opening of right B3, with improvement of the luminal stenosis.

\section{Case 2}

A 45-year-old male was evaluated for a cough, rhinorrhea, and generalized malaise. Chest X-ray showed an infiltrative shadow with a cavitary lesion, so he was admitted to hospital. His past medical history was unremarkable. On physical examination at admission, the patient was afebrile, but auscultation revealed diminished breath sounds in the left lower lung field. Blood tests showed a WBC count of 11,500 (seg $81.5 \%)$ and CRP of $20.2 \mathrm{mg} / \mathrm{dl}$. Sputum cultures revealed no significant pathogens. Chest CT (Fig. 4A) showed a lung abscess in left S10. Bronchoscopic findings (Fig. 5A) were a protruding lesion at the opening of left $\mathrm{B} 10$, and the lumen was stenosed.

The histological findings (Fig. 6) were integrity of the bronchial epithelium, bleeding in the submucosal tissue, neutrophilic and histiocytic infiltration, and changes of 

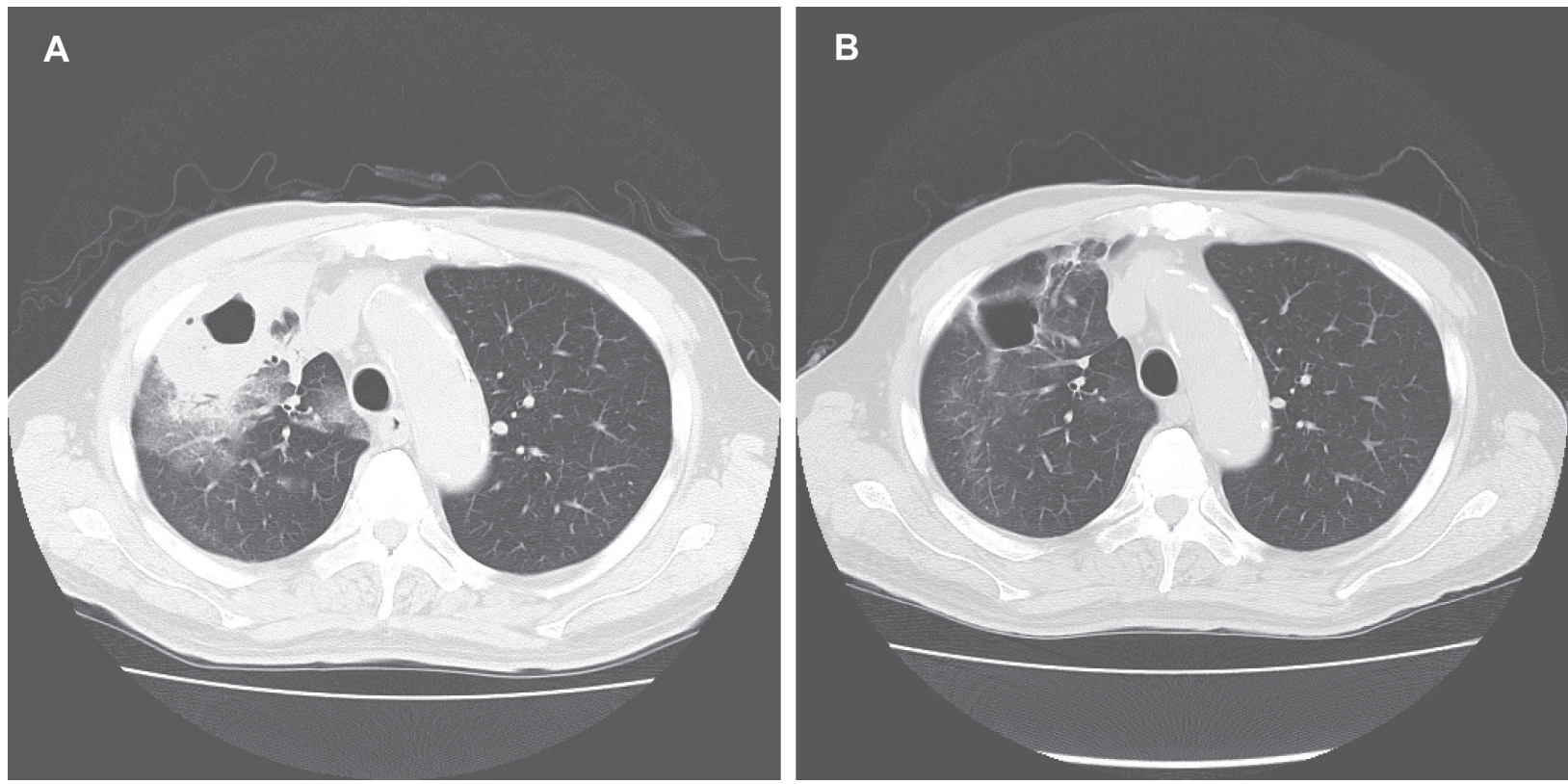

Figure 1. (A) Chest CT showed a lung abscess in right S3; (B) Chest CT showed a cavitary lesion, but the fluid accumulation resolved.

suppurative inflammation. The diagnosis was a bronchial inflammatory polyp. SBT/ABPC $6 \mathrm{~g}$ was administered by intravenous infusion for 18 days. About 5 months after treatment, Chest CT (Fig. 4B) showed improvement of the lung abscess. A bronchoscopy on day 28 (Fig. 5B) revealed no abnormal findings.

\section{Case 3}

A 65-year-old male was evaluated for a productive cough. Chest CT showed a cavitary lesion, he was admitted to hospital. His past medical history was unremarkable. The ad-
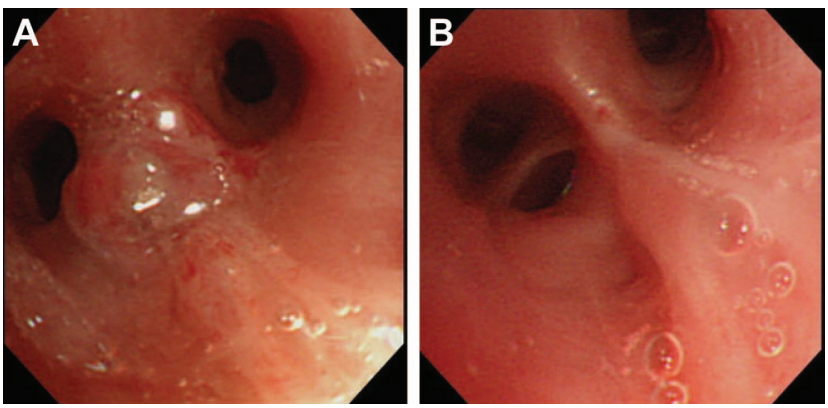

Figure 2. (A) Bronchoscopic findings was a protruding lesion at the opening of right B3, with stenosis of the involved bronchus; (B) A bronchoscopy on day 24 was disappearance of the protruding lesion at the opening of right B3, with improvement of the luminal stenosis. mission physical examination was normal except for a fever of $37.0^{\circ} \mathrm{C}$. Blood tests showed a WBC count of 11,400 (seg $64.5 \%$ ) and CRP of $5.7 \mathrm{mg} / \mathrm{dl}$. Sputum cultures revealed no significant pathogens. Chest CT (Fig. 7A) showed a lung abscess in right $\mathrm{S} 10$. Bronchoscopic findings (Fig. 8A) were a protruding lesion at the opening of right B10, and the lumen was stenosed.

The histological findings (Fig. 9) were integrity of the

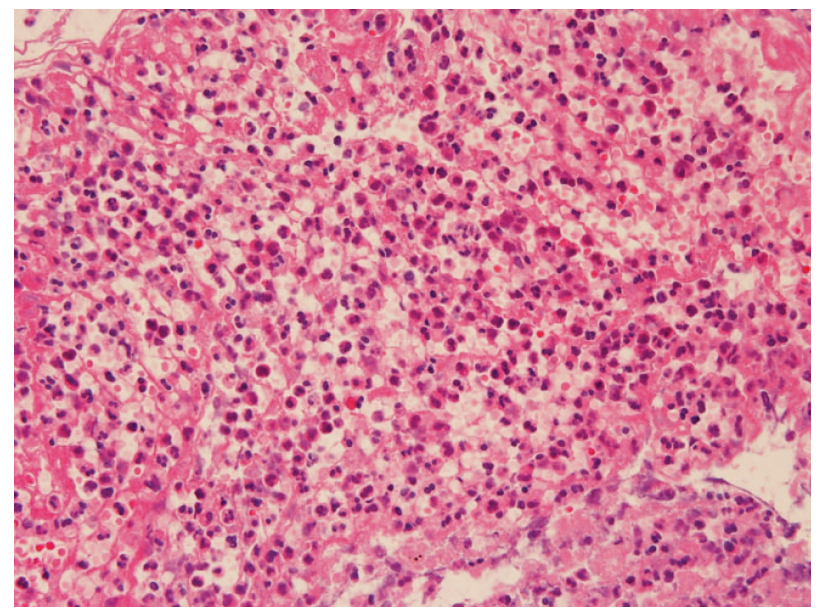

Figure 3. The histological findings obtained from bronchial biopsy were extensive neutrophilic infiltration in the submucosal tissue. In some areas, there was inflammatory cell infiltration together with fibroblast proliferation. 

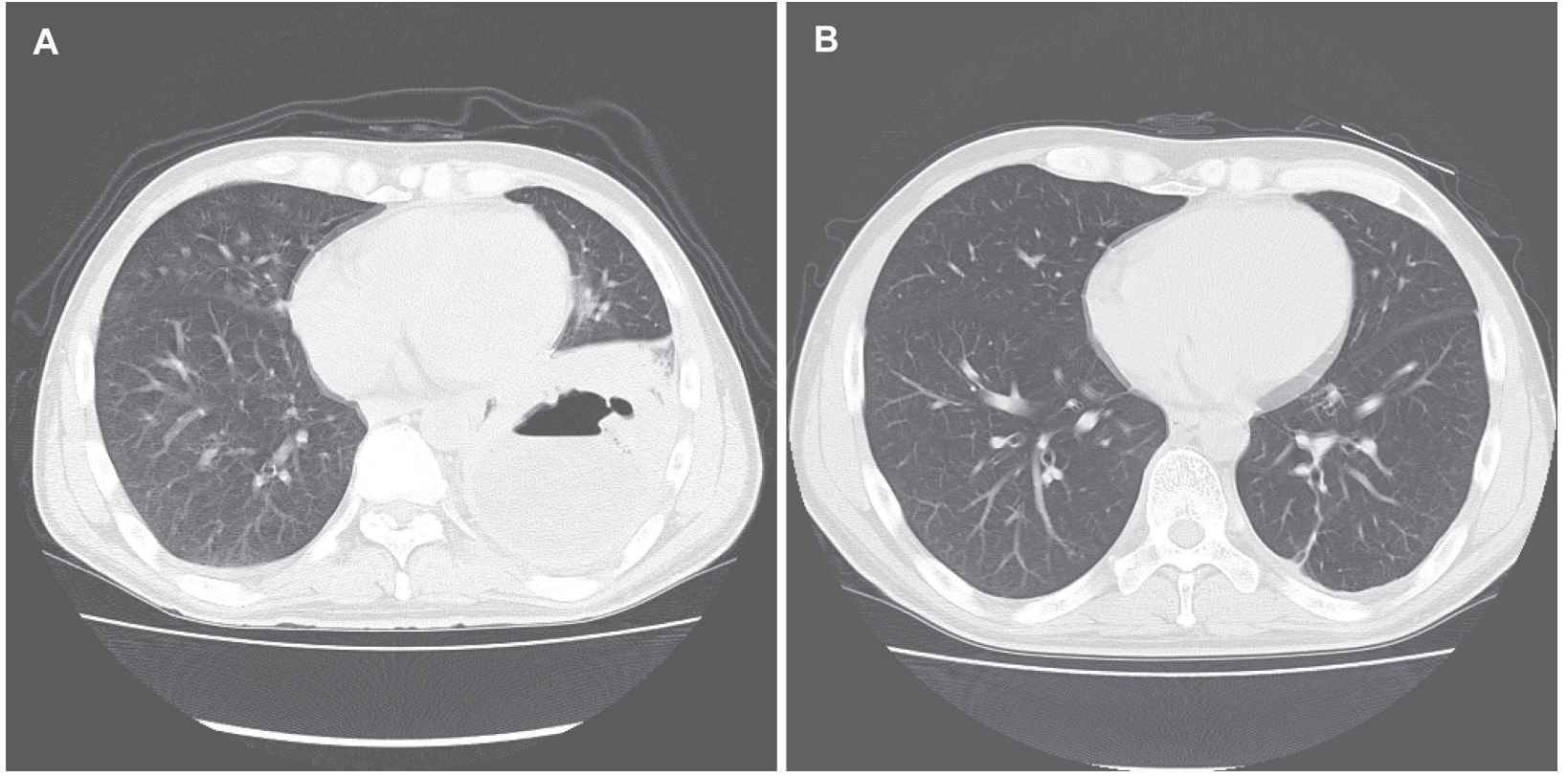

Figure 4. (A) Chest CT showed a lung abscess in left S10; (B) Chest CT showed improvement of the lung abscess.

bronchial epithelium and extensive chronic inflammatory cell infiltration, including neutrophils, in the submucosal tissue. The diagnosis was a bronchial inflammatory polyp. SBT/ABPC $6 \mathrm{~g}$ was administered by intravenous infusion for 21 days. On day 20, chest CT (Fig. 7B) showed disappearance of the cavitary lesion. A bronchoscopy on day 26 (Fig. 8B) revealed disappearance of the protruding lesion at the opening of right $\mathrm{B} 10$, with improvement of the stenosis.

\section{Discussion}

The protruding lesions of the trachea and bronchi are often malignant diseases. Benign lesions may include inflamma-
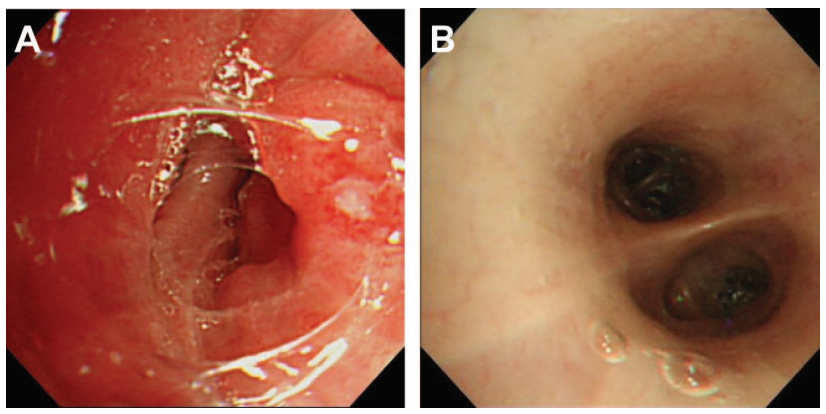

Figure 5. (A) Bronchoscopic findings were a protruding lesion at the opening of left B10, and the lumen was stenosed; (B) A bronchoscopy on day 28 revealed no abnormal findings. tory polyps, teratomas, hamartomas and lipomas, but inflammatory polyps are relatively rare [2].

The cause of inflammatory polyps, in addition to respiratory infection, includes mechanical stimulation, for example, foreign bodies, tracheostomy, or endotracheal intubation [3]. Four proposed mechanisms of bronchial inflammatory polyp formation include: stagnation of purulent secretions produced from a peripheral focus of infection, resulting in secondary inflammation of the bronchial wall [4]; primary

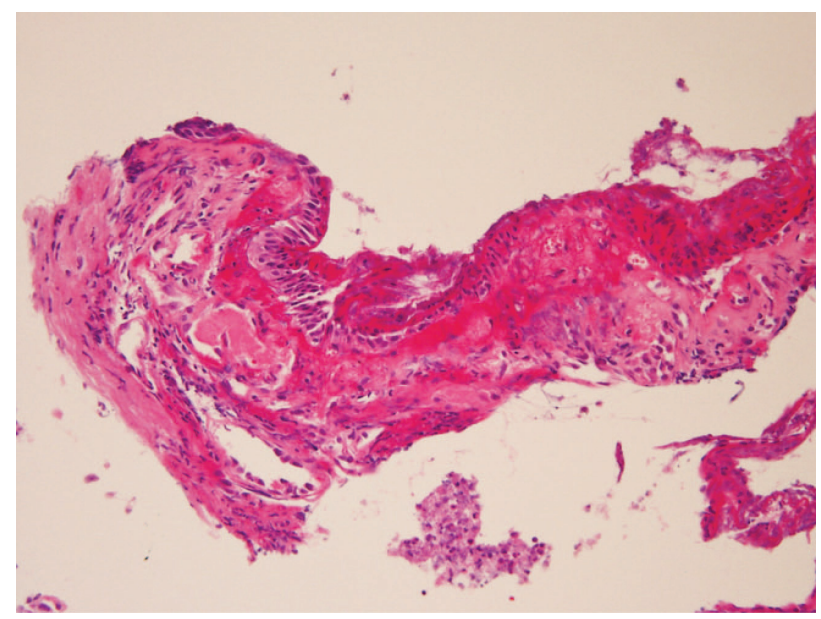

Figure 6. The historogical findings were integrity of the bronchial epithelium, bleeding in the submucosal tissue, neutrophilic and histiocytic infiltration, and changes of suppurative inflammation. 

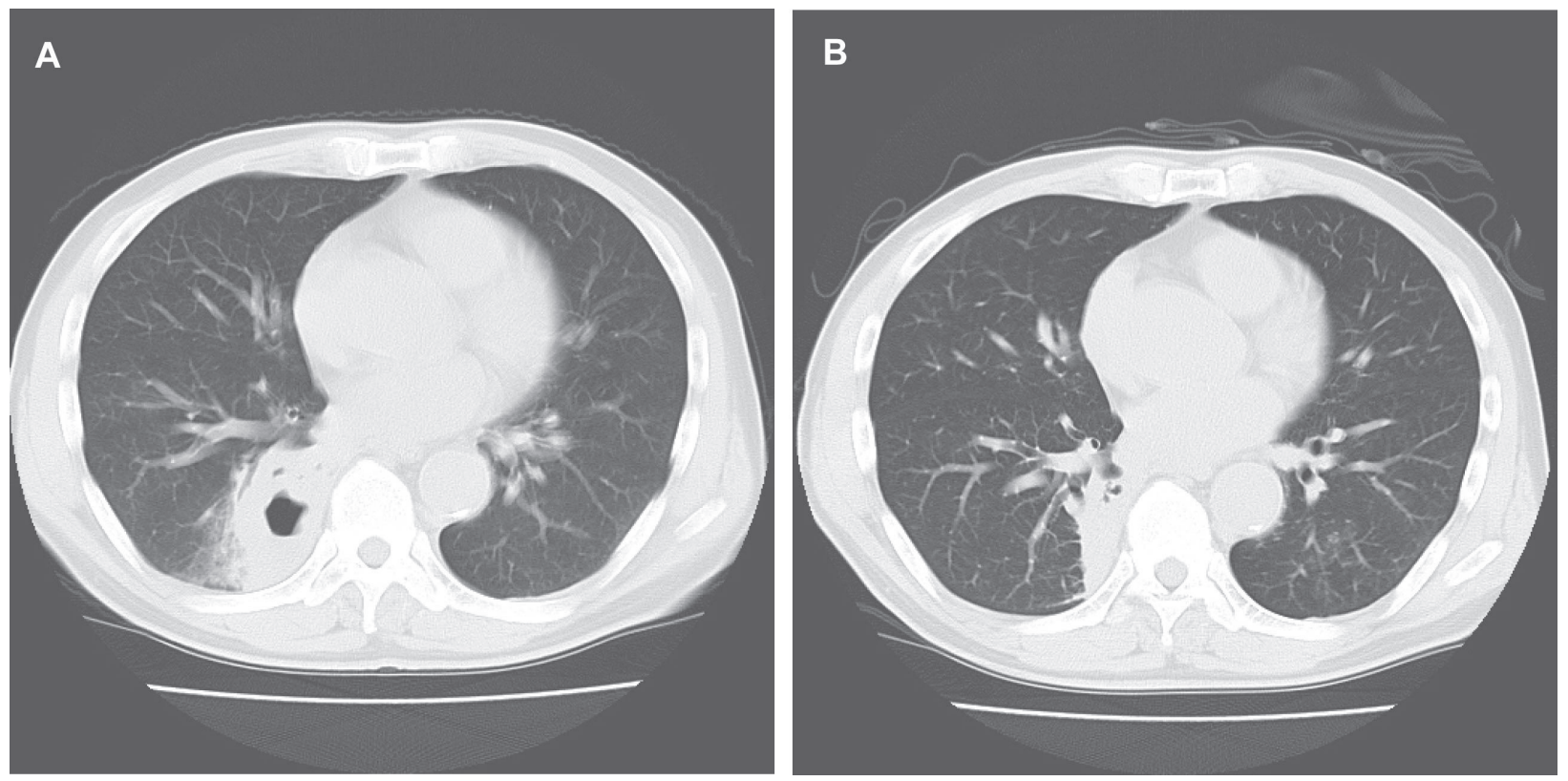

Figure 7. (A) Chest CT showed a lung abscess in right S10; (B) Chest CT showed disappearance of the cavitary lesion.

inflammation of a bronchus [5]; increased vascular permeability, leading to submucosal accumulation of exudate and mucosal protrusion [6]; and stagnant lymph flow due to localized bronchial mucosal hyperplasia and inflammation (chronic hyperplastic bronchitis) [7] .

In our three cases, histologic findings were suppurative inflammation, including neutrophilic invasion, inflammatory cells, and fibroblasts. There was no submucosal edema or hyperplasia. In addition, the polyps improved with antibiotic treatment. Therefore, this suggests stagnation of purulent secretions produced from a peripheral focus of infection associated with lung abscess formation, resulting in secondary bronchial wall inflammation and the formation of bronchial
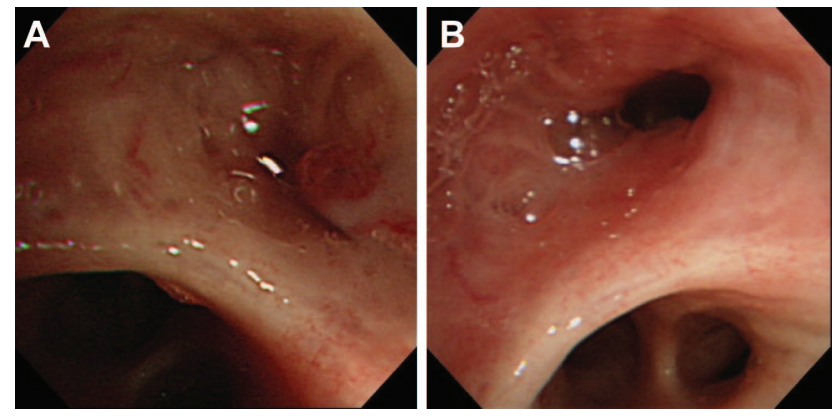

Figure 8. (A) Bronchoscopic findings were a protruding lesion at the opening of right B10, and the lumen was stenosed; (B) A bronchoscopy on day 26 revealed disappearance of the protruding lesion at the opening of right B10, with improvement of the stenosis. inflammatory polyps. This is in agreement with the mechanism proposed by Jackson et al. With malignant tumors, bronchial stenosis due to the tumor, with complications of a lung abscess from obstructive pneumonia, can occur. In our three cases, in each case, the formation of bronchial inflammatory polyps was due to secondary changes associated with a lung abscess. Bronchial stenosis by the polyps may have been a factor that increased suppuration, but it was not the cause.

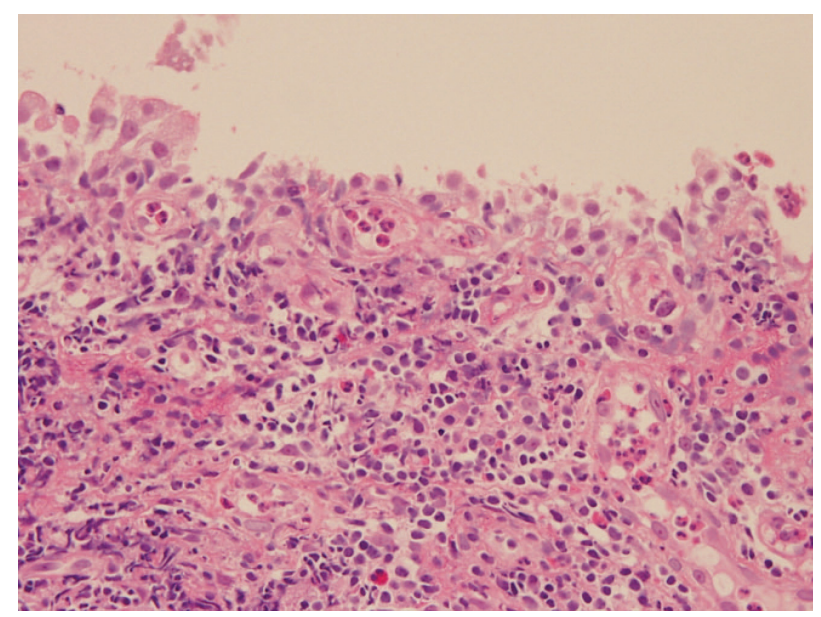

Figure 9. The historogical findings were integrity of the bronchial epithelium and extensive chronic inflammatory cell infiltration, including neutrophils, in the submucosal tissue. 
Bronchoscopy is useful to search for the cause of a lung abscess, and the protruding lesion is found in the involved bronchus. A biopsy is necessary to rule out a malignant tumor. The case of benign inflammatory polyps was found, systemic antibiotic therapy are likely to be sufficient for treatment, without the need for specific localized therapy such as resection.

Lung abscess associated with bronchial inflammatory polyps has not been previously reported. Further accumulation of such cases would be desirable.

\section{References}

1. Kawano M, Kure N, Tabeta H, Anzaki Y, Lih-fen K, Kikuchi N, Takizawa H, et al. [A case of inflammatory bronchial polyp]. Nihon Kyobu Shikkan Gakkai Zasshi 1986;24(6):698-702.
2. Ishigaki T, Tomioka S, Endo T. A Case of Inflammatory Bronchial Polyp Presenting Hemoptysis as an Initial Manifestation and Spontaneous Regression. The Japanese Journal of Chest Diseases 2001;60:1142-1147

3. Kobayashi M, Ohkubo Y, Takeyama K, Kanemura T, Aoshiba K, Takizawa T. A Case of Inflammatory Bronchial Polyp. Pharma Medica 1994;12:143-148

4. Jackson C, Jackson CL. Benign tumors of the trachea and bronchi with special reference to tumor like formations of inflammatory origin. J.M.A. 1932;99:17471754

5. Peroni A. Inflammatory bronchi. Archives of Otolaryngology 1934;19:1-21

6. Tedeschi LG, Libertini R, Conte B. Endobronchial polyp. Chest 1973;63(1):110-112.

7. Salek J, Pazderka S, Zak F. Solitary bronchial polyps of inflammatory origin; a report of two cases treated by operation. J Thorac Surg 1958;35(6):807-815. 